\title{
KiSTheIP: a program to predict thermodynamic properties and rate constants from quantum chemistry results.
}

\author{
Sébastien Canneaux ${ }^{\mathrm{a}, \mathrm{b}}$, Frédéric Bohr ${ }^{\mathrm{b}}$ and Eric Henon ${ }^{{ }^{*} \mathrm{c}}$ \\ ${ }^{[a]}$ University of Lille1 Sciences and Technologies, 59655 Villeneuve d'Ascq Cedex, France \\ ${ }^{[b]}$ LISM, EA 4695, University of Reims Champagne-Ardenne, Moulin de la Housse, Reims, 51687, \\ France \\ ${ }^{[c]}$ ICMR, UMR CNRS 7312, University of Reims Champagne-Ardenne, Moulin de la Housse, \\ Reims, 51687, France eric.henon@univ-reims.fr
}

\begin{abstract}
KiSThelP is a cross-platform free open-source program developed to estimate molecular and reaction properties from electronic structure data. To date, three computational chemistry software formats are supported (Gaussian, GAMESS, NWChem). Some key features are: gas-phase molecular thermodynamic properties (offering hindered rotor treatment), thermal equilibrium constants, transition state theory rate coefficients (TST, VTST) including one-dimensional tunnelling effects (Wigner and Eckart) and RRKM rate constants, for elementary reactions with well-defined barriers. KiSThelP is intended as a working tool both for the general public and also for more expert users. It provides graphical front-end capabilities designed to facilitate calculations and interpreting results. KiSThelP enables to change input data and simulation parameters directly through the GUI and to visually probe how it affects results. Users can access results in the form of graphs and tables. The graphical tool offers customizing of 2D-plots, exporting images and data files. These features make this program also well suited to support and enhance students learning and can serve as a very attractive courseware, taking the teaching content directly from results in molecular and kinetic modelling.
\end{abstract}




\section{Introduction}

Nowadays, theoretical chemistry is often used to estimate thermodynamic properties of molecular species such as enthalpy, entropy, heat capacity, free energy, and others through partition function calculations. Hence, by calculating free energy differences between minima of the potential energy surface (PES), equilibrium constants can be derived. Analogously, prediction of chemical reaction rate constants requires electronic quantum-mechanical calculations to obtain activation barriers. In order to determine these thermodynamic and kinetic properties from quantum results, the use of statistical mechanics is required for relating the microscopic properties of individual atoms and molecules to the macroscopic bulk properties of materials that are under study in your laboratory. For this, it is not always easy to find general software providing solution completely adapted to your needs. That is the reason why we have initially written our own code (initially called Kisthep) to compute thermodynamic, equilibrium and kinetic properties for molecular systems of atmospheric interest, from first principles. This work has resulted in the program KiSThelP (Kinetic and Statistical Thermodynamical Package) that we have built up over the years and that we present herein. A strong effort regarding the user-friendliness was made in view of its publication and use. It is intended as a working tool both for the general public and also for more expert users. It has been developed with the aim to facilitate the treatment of quantum results in order to estimate molecular and reaction properties. It is not designed to be comprehensive such as for example the polyRate ${ }^{1}$ or MultiWell $^{2-4}$ programs that include numerous features and many options for computing chemical reaction rate constants. Having a Graphical User Interface (GUI), KiSThelP is undergoing towards a tool that runs smoothly, making it easy to get first results without spending too much time with documentation. Source code is available and free, what enables adding further abilities and improvement of the program by others. Beyond equilibrium and rate constant calculations, KiSThelP allows for examining the change of molecular properties in a range of temperatures and pressures using the GUI. In this respect, KiSThelP is well suited as an introduction for students learning statistical mechanics, thermochemistry and kinetics. It is helpful to prepare data files to start calculating properties from quantum chemistry results and to analyze results. It is in this regard a teaching and research aid. The code base has been structured to be easily extendable using the object-oriented Java programming language.

In this article, the main features of KiSThelP are described. Both the implementation and usage are addressed. The paper is organized as follows: in section 2, we present the calculation and output options of KiSThelP. After that, it is explained in section 3 how 
KiSThelP processes the input file to collect the data it needs. We then introduce the main features of KiSThelP. The last section compares the possibilities offered by KiSThelP with those of other programs.

\section{General features of KiSTheIP}

KiSThelP is written in the programming language Java. One of the most significant advantages of Java is the ability to run a code on many different operating systems, thus making KiSThelP platform-independent. Since Java is object-oriented, KiSThelP has been written as a modular program and reusable code. This makes it easy to maintain, modify and extend the existing code as new objects can be created with small differences to existing ones. The reference manual is made available through the Help menu of KiSThelP both for users and developers.

KiSThelP has been designed to perform statistical mechanics calculations from ab initio quantum chemistry data, without the need of an analytical potential energy function. Quantities in the canonical and micro-canonical ensembles are calculated. This results in the computation of molecular thermodynamic properties, thermal equilibrium constants, transition state theory $\left(\mathrm{TST}^{5-7}\right)$ rate coefficients, and $\mathrm{RRKM}^{8-12}$ rate constants. Onedimensional tunnelling and variational effects have also been incorporated in the TST calculations. All these methods are widely explained in the literature and won't be addressed in more details here. For validation of inclusion of these techniques in our program we refer to our previous works ${ }^{13-16}$.

\section{Chemical species}

The equations used for computing statistical and thermochemical data in KiSThelP are from standard texts on thermodynamics. The starting point is the calculation of the partition function $\mathrm{Q}_{\mathrm{x}}(\mathrm{V}, \mathrm{T})$ for the corresponding component $\mathrm{x}$ of the total partition function. Equations used to calculate the translational, electronic, rotational and vibrational contributions in the canonical ensemble are given in Table $\mathbf{1}$.

They are equivalent to those given in standard texts on physical chemistry. ${ }^{17}$ At a given temperature, the data required to derive the partition functions $Q_{v i b}^{v=0}$ from ab initio calculations are the frequencies $\left(v_{\mathrm{i}}\right)$ of vibrational modes obtained within the harmonic oscillator approximation. In calculating the vibrational partition function, energy is measured from the ground state $(\mathrm{v}=0)$. But, we also print out $\mathrm{Q}_{\mathrm{vib}}^{\text {BOT }}$ obtained by choosing 
the zero of energy to be the bottom of the potential energy curve. Translational partition function, $\mathrm{Q}_{\text {trans }}$, only depends on the molecular mass, temperature and pressure in the ideal gas approximation. It is to be noted that the standard state of pure gaseous species (ideal gas at $\mathrm{P}^{0}=1$ bar) is used in chemical equilibrium and TST kinetic properties computations, but the molecular properties calculated with KiSThelP can also be estimated at any pressure. For molecular species, the moments of inertia derived from the geometry are used as input for calculating the rotational partition function $\mathrm{Q}_{\mathrm{rot}}$ in the rigid rotor approximation. Three cases are envisaged: single atoms $\left(\mathrm{Q}_{\text {rot }}\right.$ is set to 1$)$, linear polyatomic molecules, and general non-linear polyatomic molecules. In the equations given in Table $1, \sigma_{\mathrm{r}}$ is the rotational symmetry number. It is a factor that compensates for overcounting identical states. It must be supplied by the user and put in the input data file. Spacing of electronic energy levels is assumed to be very large compared to $\mathrm{k}_{\mathrm{b}} \mathrm{T}$ so that the excited electronic states do not make a significant contribution to the total partition function $\mathrm{Q}_{\text {elec }}$. The electronic partition function then reduces to the degeneracy of the ground state. The energy of the ground state is set to zero. As usually adopted in statistical mechanics for molecules, vibration, translation, rotation and electronic modes are assumed uncoupled. Hence, in KiSThelP, the total partition function of a molecule in its ground state is calculated as:

$$
\mathrm{Q}_{\text {tot }}=\mathrm{Q}_{\text {trans }} \times \mathrm{Q}_{\text {vib }} \times \mathrm{Q}_{\text {rot }} \times \mathrm{Q}_{\text {elec }}
$$

These statistical calculations provide the bridge between quantum mechanics of individual molecules and resulting thermodynamic properties that are reported in the KiSThelP output. Contributions to the thermodynamic properties are computed according to relations reported Table 1. It should be kept in mind that the absolute enthalpies computed from quantum mechanics are relative to infinitely separated electrons and nuclei.

The thermodynamic properties computations clearly have some limitations the user must be aware of. The Born-Oppenheimer and ideal gas approximations are invoked. The electronic partition function is truncated after the first term. The rigid-rotor approximation is assumed. Obtained thermochemical data must be used with caution since the harmonic oscillator approximation breaks down above about $1500 \mathrm{~K}$ because anharmonic effects begin to make significant contributions to the vibrational energy. In the field of gas-phase computational kinetics, the two last approximations are mainly the most problematic and attempts have been made to correct them. 
Due to the neglect of anharmonicity in vibration frequency prediction and because of approximations in the potential energy calculation, it is known that there is a systematic error in the predicted frequencies when they are compared to the experimental fundamental vibrational frequencies. A scaling factor ${ }^{18}$ is commonly used to correct the calculated value to match the experimental one. Furthermore, though the harmonic oscillator approximation provides a fast and straightforward method to determine the vibrational frequencies used to compute the partition function of internal modes, it breaks down for large-amplitude internal motions such as internal rotations. The hindered rotor approximation offers a significant improvement as compared to the harmonic oscillator treatment. That is the reason why the approach of McClurg et al. ${ }^{19}$ has been implemented in KiSThelP to account for deviations from the harmonic oscillator (HO) value. The calculations are based on two quantities $\theta$ and $r$ :

$$
\theta=\frac{\mathrm{k}_{\mathrm{b}} \mathrm{T}}{\mathrm{h} v} \quad \mathrm{r}=\frac{\Delta \mathrm{E}}{\mathrm{h} v}
$$

The deviations proposed by the authors for the vibrational contributions are:

$$
\begin{array}{r}
\Delta \mathrm{ZPE}=-\frac{\mathrm{h} v}{(2+16 \mathrm{r})} \quad \frac{\mathrm{Q}}{\mathrm{Q}_{\mathrm{HO}}}=\sqrt{\frac{\pi \mathrm{r}}{\theta}} \times \mathrm{e}^{-\frac{\mathrm{r}}{2 \theta}} \times \mathrm{I}_{0}(\mathrm{r} / 2 \theta) \\
\Delta \mathrm{U}=\mathrm{k}_{\mathrm{b}} \mathrm{T}\left[-0.5+\frac{\mathrm{r}}{2 \theta}\left(1+\frac{\mathrm{I}_{1}(\mathrm{r} / 2 \theta)}{\mathrm{I}_{0}(\mathrm{r} / 2 \theta)}\right)\right] \\
\Delta \mathrm{C}_{\mathrm{V}}=\mathrm{k}_{\mathrm{b}}\left[-0.5+\frac{\mathrm{r}^{2}}{8 \theta^{2}}\left\{1-2\left[\frac{\mathrm{I}_{1}(\mathrm{r} / 2 \theta)}{\mathrm{I}_{0}(\mathrm{r} / 2 \theta)}\right]^{2}+\frac{\mathrm{I}_{2}(\mathrm{r} / 2 \theta)}{\mathrm{I}_{0}(\mathrm{r} / 2 \theta)}\right\}\right]
\end{array}
$$

where the corrections $\Delta \mathrm{U}$ and $\mathrm{Q} / \mathrm{Q}_{\text {но }}$ exclude ZPE contribution and $\mathrm{I}_{\mathrm{n}}(\mathrm{r} / 2 \theta)$ is the Bessel function of order $\mathrm{n}$. The method used (HRDS) is based on an interpolation scheme between quantum-mechanical partition function at low temperature and the classical partition function at high temperature. The only input parameter to HRDS is the ratio of barrier height $\Delta \mathrm{E}$ to harmonic frequency. However, torsions exhibiting multiple-wells energy curves, with conformers favoured over others conformers cannot be well represented by this method.

\section{Calculation of gas-phase chemical reaction equilibrium constants}

Beyond calculations for molecular systems, KiSThelP has been designed to compute gasphase chemical reaction properties. First of all, chemical equilibrium constants can be determined ("Calculation/Equilibrium" menu). The knowledge of equilibrium constants is important in the study of chemical reaction mechanisms. In addition to allowing the prediction of the composition of a mixture, the equilibrium constant is also connected to rate constants through the existence of equilibriums between stable species in multiple- 
step processes. Furthermore, in simulations of gas-phase mixtures (atmosphere, combustion processes, chemical vapour deposition simulations, ...), backward rate can be found using the forward rate and the equilibrium constant. In other respects, the equilibrium constant is a practical indicator to test theoretical methods because it is very sensitive both to the energy quality and to geometrical parameters and also because the electronic structure of each compound that takes part in the equilibrium is extremely different from the others.

To calculate equilibrium constants one needs to calculate the associated standard reaction Gibbs energy $\Delta \mathrm{G}^{0}$ at temperature $T$, hence the Gibbs free energy for each of the species at a pressure of one bar and at temperature T. Such calculations are straightforward provided that the user supplies the values of stoichiometric numbers of the balanced chemical equation. The thermodynamic expression of the equilibrium constant for gasphase reactions is employed in KiSThelP:

$$
\mathrm{K}_{\text {eq }}=\mathrm{e}^{-\frac{\Delta \mathrm{G}^{0}(\mathrm{~T})}{\mathrm{RT}}}
$$

where $\mathrm{R}$ is the ideal gas constant. The standard state of a pure gaseous substance is used (ideal gas at one bar pressure).

\section{Theoretical prediction of rate constants for elementary gas-phase chemical reactions.}

Additionally, KiSThelP includes the theoretical prediction of canonical and microcanonical rate constants for gas-phase chemical reactions. The methods used are conventional transition state theory (TST) $)^{5,6}$ or variational transition state theory ${ }^{7}$, or RRKM theory ${ }^{8-12}$. One-dimensional quantum mechanical tunnelling treatments through Wigner correction ${ }^{20}$ or an unsymmetrical Eckart potential energy barrier ${ }^{21}$ can also be involved. All these methods are widely explained in the literature and won't be addressed in more details here. Both gas-phase unimolecular and bimolecular elementary reactions with a barrier are considered.

\section{TST}

In conventional $\operatorname{TST}^{5,6}$ the transition state is located at the first-order saddle point on the PES. Hence, TST requires information only for the saddle point and the reactant(s). The equation usually presented for the conventional TST is:

$$
\mathrm{k}^{\mathrm{TST}}(\mathrm{T})=\sigma \frac{\mathrm{k}_{\mathrm{b}} \mathrm{T}}{\mathrm{h}} \frac{\mathrm{Q}^{\mathrm{TS}}(\mathrm{T})}{\mathrm{N}_{\mathrm{A}} \mathrm{Q}^{\mathrm{R}}(\mathrm{T})} \mathrm{e}^{-\frac{\mathrm{V}^{\ddagger}}{\mathrm{k}_{\mathrm{b}} \mathrm{T}}}
$$


where $\sigma$ is the reaction path degeneracy, $k_{b}$ is the Boltzmann's constant, $T$ is the temperature, $\mathrm{h}$ is the Planck's constant, $\mathrm{N}_{\mathrm{A}}$ is Avogadro's number (it disappears for unimolecular reactions rate constants with units of $\left.\mathrm{s}^{-1}\right)$. $\mathrm{V}^{\ddagger}$ is the difference in zero-point excluded potential energy between the transition state (TS, assumed to be located at the saddle point on the PES) and the reactant(s) (zero-point energy contributions are included in the partition functions). $\mathrm{Q}^{\mathrm{TS}}$ and $\mathrm{Q}^{\mathrm{R}}$ denote the total partition functions of the TS and the reactant(s) with the translational partition functions expressed in per unit volume. $\mathrm{Q}^{\mathrm{TS}}$ excludes the reaction coordinate. The thermodynamic equivalent of Eq. (2) is employed in KiSThelP:

$$
\mathrm{k}^{\mathrm{TST}}(\mathrm{T})=\sigma \frac{\mathrm{k}_{\mathrm{b}} \mathrm{T}}{\mathrm{h}}\left(\frac{\mathrm{RT}}{\mathrm{P}^{0}}\right)^{\Delta \mathrm{n} \mathrm{e}^{-\frac{\Delta \mathrm{G}^{0, \ddagger}(\mathrm{T})}{\mathrm{k}_{\mathrm{b}} \mathrm{T}}}}
$$

where $\Delta \mathrm{G}^{\sharp, 0}(\mathrm{~T})$ represents the standard Gibbs free energy of activation for the considered reaction $(\Delta \mathrm{n}=1$ or 0 for gas-phase bimolecular or unimolecular reactions, respectively; $\mathrm{RT} / \mathrm{P}^{0}$ has the unit of the inverse of a concentration). In the calculation of $\Delta \mathrm{G}^{\ddagger 0}(\mathrm{~T})$, the imaginary frequency that corresponds to the reaction coordinate degree of freedom is removed from the vibrational partition function of transition state, and thus from the kinetic treatment (except when tunnelling correction calculation is invoked).

To determine the reaction path degeneracy $\sigma$, thinking about all the various possibilities can rapidly become confusing. For that, we recommend the articles by A.J. Karas et al. and R.G. Gilbert et al. ${ }^{22,}{ }^{23}$ When calculating reaction rate constants, KiSThelP removes the rotational symmetry numbers from all partition functions (even though this data must be present in the input files of reactants and transition state) because these numbers are already included in the reaction path degeneracy $\sigma$. The user must supply the reaction path degeneracy directly through the GUI. This number is correctly given by:

$$
\sigma=\frac{\mathrm{n}^{\ddagger} \times \sigma^{\mathrm{R}}}{\mathrm{n}^{\mathrm{R}} \times \sigma^{\ddagger}}
$$

where $\mathrm{n}$ and $\mathrm{n}^{*}$ are the number of chiral isomers of the reactant(s) and transition state, respectively, and $\sigma^{\mathrm{R}}$ and $\sigma^{\ddagger}$ are the rotational symmetry numbers. In other respects, dual level calculations, which combine properties obtained at a low-level of theory with accurate properties computed at a high-level, can be carried out by changing properties values directly in the KiSTHelP input files.

\section{Tunnelling effect}

In KiSThelP, one-dimensional quantum mechanical effects on reaction coordinate motion are incorporated by a multiplicative transmission coefficient $\chi(\mathrm{T})$, so that: $\mathrm{k}^{\mathrm{TST} / \mathrm{T}}(\mathrm{T})=\chi(\mathrm{T})$ 
$\times \mathrm{k}^{\mathrm{TST}}(\mathrm{T})$, where TST/T denotes TST completed with tunnelling corrections. In a first approach, the so-called Wigner correction ${ }^{20}$ can be applied, only based on the imaginary frequency $\operatorname{Im}\left(v^{\star}\right)$, which characterizes the reaction mode through the relation:

$$
\chi(\mathrm{T})=1+\frac{1}{24}\left(\frac{\mathrm{h} \operatorname{Im}\left(v^{\ddagger}\right)}{\mathrm{k}_{\mathrm{b}} \mathrm{T}}\right)^{2}
$$

Another approach is proposed in KiSThelP to compute this multiplicative factor that is a tunnelling treatment involving an unsymmetrical Eckart potential energy barrier. ${ }^{21}$ This methodology requires no ab initio calculations at points other than reactants, products, and saddle point. User will be asked for the ZPE corrected reverse energy barrier. The analytic form proposed by Eckart can model a variety of physically reasonable shapes (involving unsymmetrical forms) and admits an analytical solution of the corresponding Schrödinger equation and then for the probability $\mathrm{p}(\mathrm{E})$ of transmission through the corresponding 1-D barrier at energy E. Original formula of Eckart are used in KiSThelP with $\mathrm{L}$, the width of the transition region which can be defined in term of the imaginary frequency $\operatorname{Im}\left(v^{\star}\right)^{9}$ characterizing the reaction mode:

$$
\begin{gathered}
\mathrm{p}(\mathrm{E})=1-\left[\frac{\cosh [2 \pi(\alpha-\beta)]+\cosh [2 \pi \delta]}{\cosh [2 \pi(\alpha+\beta)]+\cosh [2 \pi \delta]}\right] \\
\alpha=\frac{1}{2 \sqrt{\mathrm{C}}} \sqrt{\mathrm{E}} \quad \beta=\frac{1}{2 \sqrt{\mathrm{C}}} \sqrt{\mathrm{E}-\mathrm{A}} \delta=\frac{1}{2 \sqrt{\mathrm{C}}} \sqrt{\mathrm{B}-\mathrm{C}} \\
\mathrm{A}=\Delta \mathrm{H}_{\mathrm{f}}^{\ddagger, \mathrm{KK}}-\Delta \mathrm{H}_{\mathrm{r}}^{\ddagger, \mathrm{KK}} \\
\mathrm{B}=\left(\sqrt{\Delta \mathrm{H}_{\mathrm{f}}^{\ddagger}, \mathrm{KK}}-\sqrt{\Delta \mathrm{H}_{\mathrm{r}}^{\ddagger}, \mathrm{KK}}\right)^{2} \\
\mathrm{C}=\left(\mathrm{h} \operatorname{Im}\left(v^{\ddagger}\right)\right)^{2}\left[\frac{\mathrm{B}^{3}}{\left(\mathrm{~A}^{2}-\mathrm{B}^{2}\right)}\right]^{2}
\end{gathered}
$$

The two constants (A, B) determining the overall shape of the Eckart barrier are directly linked to the zero-point corrected energy barriers in the reverse and forward direction computed by KiSThelP: $\Delta \mathrm{H}_{\mathrm{r}}^{\ddagger, 0}$ and $\Delta \mathrm{H}^{\ddagger}{ }_{\text {f }}$. Finally, the Eckart tunnelling correction $\chi(\mathrm{T})$ is obtained by numerically integrating $\mathrm{p}(\mathrm{E})$ over a Boltzmann distribution of energies:

$$
\chi(T)=\frac{e^{\frac{\Delta H_{f}^{\ddagger, 0 K}}{k_{b} T}}}{k_{b} T} \int_{0}^{\infty} p(E) e^{-\frac{E}{k_{b} T}} d E
$$

In KiSThelP, the algorithm uses a 15-point Gauss-Laguerre integration. In the case of an endothermic reaction, roots of 15 th degree Laguerre polynomial are distributed on an 
energy range above A. The Gauss-Laguerre scheme can still be used by means of an integration variable change, leading to:

$$
\chi(T)=\frac{e^{\frac{\Delta H_{r}^{\ddagger, 0 K}}{k_{b} T}}}{k_{b} T} \int_{0}^{\infty} p(E+A) e^{-\frac{E}{k_{b} T}} d E
$$

However, the potential energy along IRC has not always a shape allowing tunnelling probability to be properly assessed by an Eckart potential. Furthermore, although convenient to carry out and thus attractive, these methods (Wigner and Eckart) do not take into account the full dimensionality of the quantum effect. One of the possible extensions of KiSThelP would be to program the semi-classical WKB approximation in order to better estimate the permeability $\mathrm{P}(\mathrm{E})$, using zero curvature tunnelling (ZCT, which does not include corner-cutting tunnelling) or event more accurate small-curvature tunnelling (SCT) or large curvature tunnelling (LCT) approaches. ${ }^{7}$

\section{VTST}

Available in KiSThelP, the canonical variational transition state theory (CVT) involves a generalized transition state $^{24}$ rate constant $\mathrm{k}^{\mathrm{GT}}$ :

$$
\mathrm{k}^{\mathrm{GT}}(\mathrm{T})=\sigma \frac{\mathrm{k}_{\mathrm{b}} \mathrm{T}}{\mathrm{h}} \frac{\mathrm{Q}^{\mathrm{TS}}(\mathrm{T}, \mathrm{s})}{\mathrm{N}_{\mathrm{A}} \mathrm{Q}^{\mathrm{R}}(\mathrm{T})} \mathrm{e}^{-\frac{\mathrm{V}^{\ddagger}(\mathrm{s})}{\mathrm{k}_{\mathrm{b}} \mathrm{T}}}
$$

where $s$ is the distance along the minimum energy reaction path (MEP) in iso-inertial coordinates. In this theory $(\mathrm{CVT})$, the rate constant $\mathrm{k}^{\mathrm{CVT}}(\mathrm{T})$ is obtained by minimizing $\mathrm{k}^{\mathrm{GT}}$ with respect to $\mathrm{s}$ along the reaction path:

$$
\mathrm{k}^{\mathrm{CVT}}=\min _{\mathrm{s}}^{\mathrm{GT}}(\mathrm{T}, \mathrm{s})
$$

The thermodynamic equivalent of equation (15) is employed in KiSThelP $\left(\mathrm{k}^{\mathrm{CVT}}(\mathrm{T})\right.$ is obtained by minimizing the generalized free energy of activation $\left.\Delta \mathrm{G}^{\ddagger, 0}(\mathrm{~T})^{\mathrm{GT}}\right)$. Addition information is needed on the PES compared to conventional TST. The MEP can be obtained by performing a so-called intrinsic reaction path ${ }^{25}$ (IRC) calculation using a quantum chemistry software. By convention, $\mathrm{s}=0$ at transition state structure, $\mathrm{s}<0$ on reactant side and $s>0$ on product size. In VTST, the required information is the MEP information obtained from electronic structure calculations. This information is read from a reaction path KiSThelP input file containing as "POINT" sections as points calculated along the reaction path (including the transition state). The coordinate reaction, potential energy, inertia moment, ... (see KiSThelP documentation) must be supplied for each point. Although such a definition of the generalized TS would allow dealing with loose TS, KiSThelP is more appropriate for elementary reactions with a well-defined first-order saddle point on the potential energy surface (tight TS). Treating reactions involving a 
loose TS or barrier-less reactions where two fragments have nearly free internal rotations in a dissociation/recombination mechanism needs more elaborated treatments. In particular, it requires optimizing not only the value of the reaction coordinate but also its definition. ${ }^{26,27}$

Along the reaction path, ideally, all bound modes transverse to the reaction path should be characterized by no imaginary frequency in KiSThelP. But practically, from electronic structure calculations, a reaction path point can have 0,1 , or more than one imaginary boundary modes normal to the reaction mode. In KiSThelP, 0 or 1, or more imaginary frequencies will be accepted for a reaction path point. But if 0 imaginary frequency is found, then, to handle a homogeneous set of data the smallest frequency is set to imaginary number to design the reaction mode (thus it is removed from statistical and kinetics treatments). If more than one imaginary frequency are found, corresponding imaginary frequencies are removed from statistical treatments. In all these cases, warnings are delivered to the user.

\section{RRKM}

Unimolecular processes in gas phase can also be addressed on a micro-canonical basis and pressure effects (falloff behaviour) can be accounted for by RRKM equations. The implementation of this approach in KiSThelP is based on the books by K.A. Holbrook et al., ${ }^{11}$ P. J. Robinson et al. ${ }^{10}$ and W. Forst. ${ }^{9}$ Only reaction paths with a well-defined firstorder saddle point on the potential energy surface are considered in KiSThelP (tight TS).

The energy-dependent micro-canonical rate constants $\mathrm{k}(\mathrm{E})$ are calculated by the standard RRKM expression:

$$
\mathrm{k}(\mathrm{E})=\frac{\sigma \mathrm{G}(\mathrm{E})}{\mathrm{h} \mathrm{N}(\mathrm{E})}
$$

where $\sigma$ is the reaction path degeneracy, $G(E)$ is the total number of states of the transition state with energy less than or equal to $\mathrm{E}$, and $\mathrm{N}(\mathrm{E})$ is the density of states of the dissociating reactant species. The reactant and transition state are approximated as symmetric tops and the external K-rotor, associated with the smallest moment of inertia, is treated as an active degree of freedom completely coupled to the vibrations. In KiSThelP, Laplace-transform method based on the inversion of the partition functions is employed for the calculation of the sum of states $G(E)$ and density of states $N(E)$. The thermal rate constant is obtained by using the following expression: 


$$
k(T)=\frac{\sigma Q_{1}^{+}}{Q_{2} Q_{1} h} e^{-\frac{E_{0}}{k_{b} T}} \int_{0}^{\infty} \frac{G(E) e^{-\frac{E}{k_{b} T}}}{1+\frac{k\left(E_{0}+E+<\Delta E_{j}>\right)}{\omega}} d E
$$

where $\mathrm{h}$ is the Planck's constant, $\mathrm{Q}_{2}$ is the partition function of the active degrees of freedom (vibrations $+\mathrm{K}$-rotor) of the reactant, $\mathrm{Q}_{1}^{\ddagger}$ and $\mathrm{Q}_{1}$ are the partition functions for adiabatic rotations of the transition state and of the reactant, respectively, $\mathrm{E}_{0}$ is the zeropoint corrected threshold energy. The strong collision approximation is used assuming that every collision deactivates with $\omega=\beta_{\mathrm{c}} Z_{\mathrm{LJ}}[\mathrm{M}]$ being the effective collision frequency where $\beta_{\mathrm{c}}$ is the collisional efficiency, $\mathrm{Z}_{\mathrm{LJ}}$ is the Lennard-Jones collision frequency and $[\mathrm{M}]$ is the total gas concentration. $\mathrm{Z}_{\mathrm{LJ}}$ are calculated using the Lennard-Jones parameters: $\varepsilon / \mathrm{k}_{\mathrm{b}}(\mathrm{K})$ and $\sigma(\mathrm{cm})$. In order to account for the centrifugal effect in the rate constant calculation, the factor $\mathrm{Q}_{1} / \mathrm{Q}_{1}$ was included in the expression of $\mathrm{k}(\mathrm{T})$ and $\mathrm{k}(\mathrm{E})$ is evaluated at the energy $\mathrm{E}$ including $<\Delta \mathrm{E}_{\mathrm{j}}>$ with $\left.<\Delta \mathrm{E}_{\mathrm{j}}\right\rangle=\left(1-\mathrm{I}^{\ddagger} / \mathrm{I}\right) \times \mathrm{k}_{\mathrm{b}} \mathrm{T}$ where $\mathrm{I}^{\star}$ and $\mathrm{I}$ are the average of the two largest moments of inertia. This is to assess the possible release (or uptake) of a certain amount of the rotational energy associated with the external twodimensional inactive rotation $(\mathrm{J})$ into the active degrees of freedom as the system approach the transition state. The thermal rate constant is obtained using a 15-point Gauss-Laguerre integration. Neither variational theory nor tunnelling effect have been implemented in the present version of KiSThelP at the RRKM level.

\section{Graphical User Interface of KiSTheIP}

An easy-to-use graphical interface can be of tremendous assistance in gathering and interpreting data, and also offers a valuable educational tool. KiSThelP is tuned to provide an interactive and visually intuitive interface even on computers with low capabilities so that the program can run on standard personal computers, or be used in practical training rooms for educational purpose. Documentation is provided through the "Help" menu.

\section{Chemical species}

In order to perform a KiSThelP calculation, the user must prepare an ASCII input file (with file extension "kinp"). But alternatively, KiSThelP can read the needed electronic structure data directly from a computational chemistry software output file (this is the easiest way). Gaussian ${ }^{28}$ (g03 and g09 versions), GAMESS(2012) ${ }^{29}$ and NWChem(6.0) ${ }^{30}$ output files are supported. Frequency job output is required in order to fetch data needed to statistical computations. In this case, our program automatically builds the relevant "kinp" file. 
KiSThelP typically processes the input file to obtain keywords that tell what are the data. The content of this text file is divided into several different sections as shown on Figure 1. Each section begins with a specific keyword and ends with the *END keyword. They represent the categories of input data and are self-explanatory. For a molecular calculation, seven sections are required: MASS, FREQUENCIES, NUMBER OF SYMMETRY, LINEAR, MOMENT OF INERTIA, POTENTIAL ENERGY, ELECTRONIC DEGENERACY. For an atom only three sections are expected: MASS, POTENTIAL ENERGY, ELECTRONIC DEGENERACY. Each KEYWORD must start the line. Comments outside a *KEYWORD ... *END section are allowed, or even after the KEYWORD (on the same line). Note that keywords are not case-sensitive. But no blank lines are expected within a section. Atomic coordinates are not required. The sections can appear in any arbitrary order in the input file, however, all sections must be present.

Once the data are imported in KiSThelP for a single species, a lot of properties are delivered at the default temperature (298K) and pressure (1 bar) as illustrated in Figure 2.

Single temperature and pressure values can be interactively changed to probe their influence on properties. Data are immediately re-computed. In addition to these results, the Maxwell-Boltzmann distribution $\left(\mathrm{N}_{\mathrm{i}} / \mathrm{N}\right)=\mathrm{g}\left(\mathrm{E}_{\mathrm{i}}\right) \exp \left(-\mathrm{E}_{\mathrm{i}} / \mathrm{k}_{\mathrm{b}} \mathrm{T}\right) / \mathrm{Q}$ is plotted as a function of $\left(\mathrm{E}_{\mathrm{i}} / \mathrm{k}_{\mathrm{b}} \mathrm{T}\right)$ (bottom of Figure 2). In the case of a temperature range or a pressure range, 2D-plot can be displayed for selected properties (right panel of Figure 3). The graphical tool offers several facilities: scales settings, changing titles $\mathrm{x} / \mathrm{y}$ limits, exporting image (png format), saving numerical data $\ldots$

Also, KiSThelP is an interactive program that allows the user to change the input data directly through the GUI (left panel of Figure 2). For single molecular system calculations, the user can interactively test the influence of the mass, potential energy, electronic degeneracy, rotational symetry number, inertia moment, vibrational frequencies, on statistical and thermodynamical properties. The "vibrational" scaling factor can be changed as well. KiSThelP also enables changing temperature and pressure units during the session. Results or modified data can be stored from GUI. A session can be saved to a KiSThelP restart file and then, sometime later, be reloaded. All these features simplify data interpretation and also make this program well suited to support and enhance students learning of thermodynamics fundamentals, taking the teaching content directly from results in molecular modelling. 


\section{TST based reaction rates}

Similarly, reaction properties can be easily obtained (see Figure 4) using two input files (unimolecular reaction) or three input files (bimolecular reaction) and selecting a kinetic theory level. Concerning the TS (or a minimum energy path), a specific input format is required. The corresponding file is referred as the "reaction path kinp" file in the documentation. This file wraps the reaction path points information (including TS) in several “**POINT ...**END” sections. This new section type gathers molecular keywords together with a new IRC section. Facilities are provided by KiSThelP to build this specific reaction path "kinp" file (menu "Data"). This tool concatenates molecular individual "kinp" files corresponding to each reaction path point and adds appropriate IRC information (interactively supplied by the user). Thus, in order to start a TST calculation from quantum chemistry results as input, the user must first generate as many "kinp" files as points on the reaction path (only one for the TS in a standard TST calculation).

When a VTST calculation is required, the potential energy profile is plotted and compared to the free energy profile to emphasize the variational effect on the location of the transition state at the given temperature (bottom panel of Figure 4). In addition to examine the dependence of some well-known reaction properties on temperature and pressure, the logarithmic dependence of computed rate constants can be very easily illustrated as shown in Figure 5. By choosing the VTST approach including tunnelling effect one can then compare several levels of theory on the same plot.

Commonly, temperature dependency of the rate constant can be presented using the twoand three-parameters conventional Arrhenius equations:

$$
k=A e^{-\frac{E_{a}}{R T}} k=A \times T^{n} e^{-\frac{E_{a}}{R T}}
$$

With $\mathrm{A}$ and $\mathrm{E}_{\mathrm{a}}$ the pre-factor and activation energy, respectively. This provides a quick and convenient way of predicting the temperature dependence of the rate constant values in the selected range. KiSThelP performs two statistical fittings of these two equations to the predicted data. The corresponding plots are compared to the original theoretical curves. Numerical and graphical results can be saved through the plot tool.

In addition to all the previous (numerical, graphical) results, selected micro-canonical rate constants $\mathrm{k}(\mathrm{E})$ for an unimolecular reaction are displayed as a function of $\mathrm{E} / \mathrm{k}_{\mathrm{b}} \mathrm{T}$.

\section{RRKM calculations}


In addition to the two input files for reactant and TS, the user will be asked for collision parameters: molecular mass (buffer gas), $\varepsilon / \mathrm{k}_{\mathrm{b}}$ (in $\mathrm{K}$ ) and $\sigma(\mathrm{cm})$ for reactant and buffer gas respectively. These data will serve to estimate the deactivation rate constant within the RRKM unimolecular decomposition mechanism. A small database giving $\varepsilon / \mathrm{k}_{\mathrm{b}}$ and $\sigma$ for more than 80 species is made available to users from the menu "Data".

In addition to properties previously described, major results of the RRKM treatment are also reported on the KiSThelP output as illustrated by Figure 6. Deactivation rate constant, low- and high-pressure limits $\left(\mathrm{k}^{0}\right.$ and $\left.\mathrm{k}^{\infty}\right)$ as well as $\mathrm{k}(\mathrm{RRKM})$ are given. For the 15 roots of the Laguerre polynomial, numerical values of $G(E), N(E)$ and $k(E)$ are given. In addition, the $\mathrm{k}(\mathrm{E})$ plot is displayed for energy values up to $100 \mathrm{k}_{\mathrm{b}} \mathrm{T}$.

The falloff curve (Figure 7) can be examined using the pressure range button. Then, the user can interactively test the effect of changing the collision efficiency and critical energy $E_{0}$ values on the thermal unimolecular rate constant behaviour. This last possibility can be attractive to use established high-pressure parameters to calculate the pressure dependence of the rate coefficient.

\section{Comparison with other software products}

There are not a vast number of programs that enable prediction of kinetic properties of gas-phase chemical reactions. To calculate the pressure and temperature dependence of an unimolecular process by using RRKM theory and a numerical solution of the master equation for multiple reaction channels is the main objective of some of these programs, such as MultiWell ${ }^{2-4}$, MesMer ${ }^{31}$, UNIMOL $^{32}$, VariFlex ${ }^{33}$ and ChemRate. ${ }^{34}$ Other programs, mainly centred on the use of the TST, such as POLYRATE ${ }^{1}$ and TheRate ${ }^{35}$, focus only on the temperature dependence of canonical rate constants (at high-limit pressure) but for unimolecular and bimolecular reactions. In this case, much of the effort is devoted to describe as accurately as possible quantum mechanical effects on reaction coordinate motion, using multidimensional tunnelling corrections, and to account more accurately for recrossing effects. Recent developments of the VTST extends Multistructural-VTST ${ }^{36}$ to incorporate multiple paths in the calculation of the tunnelling transmission coefficient and the variational effect: MP-VTST ${ }^{37}$, but at the expense of requiring substantial additional electronic structure information. 
Table 2 gives an overview of the capabilities of KiSThelP compared to a selection of programs among the most widely used in the field. It is to be noticed that, other programs not so well known are however worthy of attention, like for instance the recent package OpenSOAMS $^{38}(\mathrm{C}++$ library designed to compute thermodynamic data, reaction rates from first principle results) or GPOP ${ }^{39}$ (Gaussian post-processor for the estimation of thermodynamics and rate constants for gas-phase compounds and reaction, coupled to UNIMOL for RRKM calculations).

The current version of KiSThelp is theoretically less advanced than programs reported in this table. In particular, the multidimensional description of quantum effects on the reaction mode as well as mechanisms involving multiple channels are missing. Only the MultiWell ${ }^{2}$ program possesses such wide capabilities. KiSThelP exploits features of the java object-oriented language so that to enable future developments with more sophisticated workflows for applications such as master equation calculations within a multiple channel mechanism, or multiple structures TST calculations.

However, our first objective was to enable non-expert users to perform equilibrium constant, TST, VTST and RRKM calculations without to be familiar with a large number of input keywords. Nowadays, more powerful computers and more user-friendly computational tools allow more users, sometimes unfamiliar with molecular modelling, to undertake rate constant studies with vibrational and rotational constants obtained by means of quantum calculations. If the purpose is not to predict very accurate rate constants, but instead to gain early insight into a research project, use of simpler software is usually sufficient. KiSThelP is a good first step before to take up programs more sophisticated such as those abovementioned and it can be a trade-off between ease of use and predictive accuracy. Bearing this in mind, KiSThelP has got some advantages. Its GUI interface supports several file format of computational chemistry software, including one of the most popular (Gaussian). It provides graphical front-end capabilities designed to facilitate kinetic calculations and their exploitation. It can handle unimolecular and bimolecular reaction, equilibrium constant calculations, as well as temperature and pressure effects.

\section{Conclusions and Future work}

We have developed the KiSThelP program to perform kinetic, statistical and thermodynamic calculations on chemical systems and reactions. KiSThelP offers a range of features that can be helpful for experimental scientists as well as for users more 
experienced in computational kinetics. Some key features are: gas-phase molecular thermodynamic properties, thermal equilibrium constants, transition state theory rate coefficients (TST, VTST) including one-dimensional tunnelling effects, RRKM rate constants, for elementary reactions with well-defined barriers. It provides graphical frontend capabilities designed to facilitate gathering input data (data can be read directly from electronic structure files), calculations and interpreting results. A large number of parameters can be changed to interactively and visually probe their influence on predicted properties. These features make this program well suited to support and enhance students learning and can serve as a very attractive courseware as well, taking the teaching content directly from results in molecular and kinetic modelling.

The code has been structured to be easily extendable using the object-oriented Java programming language. KiSThelP is cross-platform and can be run on a standard laptop with modest hardware requirements.

Enhancements are planned for future versions of KiSThelP. Among these are: to approximate one-dimensional tunnelling by employing a user-defined potential and according to a semi-classical WKB method, to incorporate tunnelling effect in microcanonical rate constant calculations, to build 3D-surface representation of the RRKM rate constant as a function of both temperature and pressure, to propose a more comprehensive workflow with the aim of handling multiple reaction channels.

\section{Availability}

KiSThelP is available free of charge on a simplified open source license. An executable jar file, together with the source code are available at http://kisthelp.univ-reims.fr.

\section{Acknowledgments}

The authors acknowledge W. Forst, F. Caralp, N. Sokolowski-Gomez, T. Cours and J-C. Boisson for helpful discussions.

\section{Keywords}

Quantum chemistry, Statistical mechanics, Thermochemistry, Rate constant, TST, RRKM 


\section{References}

1. J. Zheng, S. Zhang, B. J. Lynch, J. C. Corchado, Y.-Y. Chuang, P. L. Fast, W.-P. Hu, Y.-P. Liu, G. C. Lynch, K. A. Nguyen, et al. POLYRATE, University of Minnesota: Minneapolis, version 2010-A 2010, Available at: http://comp.chem.umn.edu/polyrate/.

2. J. R. Barker, Int. J. Chem. Kinet. 2001, 33, 232-245.

3. J. R. Barker, Int. J. Chem. Kinet. 2009, 41, 748-763.

4. J. R. Barker, N. F. Ortiz, J. M. Preses, L. L. Lohr, A. Maranzana, P. J. Stimac, N. T.

L., T. J. Dhilip Kumar MultiWell, University of Michigan, 2011.1 2011, Available at: http://aoss.engin.umich.edu/multiwell/.

5. H. Eyring, Chem. Rev. 1935, 17, 65-77.

6. D. G. Truhlar, B. C. Garrett, S. J. Klippenstein, J. Phys. Chem. 1996, 100, 1277112800 .

7. A. Fernandez-Ramos, B. A. Ellingson, B. C. Garrett, D. G. Truhlar in Rev. Comput. Chem., 2007; Vol. 23, p. 125.

8. W. Forst, J. Phys. Chem. 1972, 76, 342-348.

9. W. Forst, in Unimolecular Reactions. A Concise Introduction C. U. Press Cambridge, 2003.

10. P. J. Robinson, K. A. Holbrook, in Unimolecular Reactions; L. N. Y. WileyInterscience 1972.

11. K. A. Holbrook, M. J. Pilling, S. H. Robertson, in Unimolecular Reactions; Wiley, Chichester, 1996.

12. R. G. Gilbert, S. C. Smith, in Theory of Unimolecular and Recombination

Reactions; Blackwell Scientific, Oxford, 1990.

13. F. Bohr, E. Henon, J. Phys. Chem. A 1998, 102, 4857-4862.

14. E. Henon, F. Bohr, J. Mol. Struct.: THEOCHEM 2000, 531, 283-299.

15. E. Henon, F. Bohr, N. Sokolowski-Gomez, F. Caralp, Phys. Chem. Chem. Phys. 2003, 5, 5431-5437.

16. F. Caralp, W. Forst, E. Henon, A. Bergeat, F. Bohr, Phys. Chem. Chem. Phys. 2006, 8, 1072-1078.

17. R. A. Alberty, R. J. Silbey, in Physical Chemistry; John Wiley \& Sons, Inc, 1992.

18. S. G. Andrade, L. C. S. Gonçalves, F. E. Jorge, J. Mol. Struct.: THEOCHEM 2008, $864,20-25$.

19. R. B. McClurg, R. C. Flagan, W. A. Goddard III, J. Chem. Phys. 1997, 106, 66756680.

20. E. Wigner, Z. Phys. Chem. B 1932, 19, 203.

21. C. Eckart, Phys. Rev. 1930, 35, 1303.

22. A. J. Karas, R. G. Gilbert, M. A. Collins, Chem. Phys. Lett. 1992, 193, 181-184.

23. A. Fernández-Ramos, B. A. Ellingson, R. Meana-Pañeda, J. M. C. Marques, D. G. Truhlar, Theor. Chem. Account 2007, 118, 813.

24. B. C. Garrett, D. G. Truhlar, J. Phys. Chem. 1979, 83, 1079-1112.

25. K. Fukui, Acc. Chem. Res. 1981, 14, 363-368.

26. S. J. Klippenstein, J. Chem. Phys. 1992, 96, 367-371.

27. S. J. Klippenstein, J. Phys. Chem. 1994, 98, 11459-11464.

28. M. J. Frisch, G. W. Trucks, H. B. Schlegel, G. E. Scuseria, M. A. Robb, J. R. Cheeseman, G. Scalmani, V. Barone, B. Mennucci, G. A. Petersson, H. Nakatsuji, M. Caricato, X. Li, H. P. Hratchian, A. F. Izmaylov, J. Bloino, G. Zheng, J. L. Sonnenberg, M. Hada, M. Ehara, K. Toyota, R. Fukuda, J. Hasegawa, M. Ishida, T. Nakajima, Y. Honda, O. Kitao, H. Nakai, T. Vreven, J. Montgomery, J. A., J. E. Peralta, F. Ogliaro, M. Bearpark, J. J. Heyd, E. Brothers, K. N. Kudin, V. N. Staroverov, R. Kobayashi, J. Normand, K. Raghavachari, A. Rendell, J. C. Burant, S. S. Iyengar, J. Tomasi, M. Cossi, N. Rega, J. M. Millam, M. Klene, J. E. Knox, J. B. Cross, V. Bakken, C. Adamo, J. Jaramillo, R. Gomperts, R. E. Stratmann, O. Yazyev, A. J. Austin, R. Cammi, C. Pomelli, J. W. Ochterski, R. L. Martin, K. Morokuma, V. G. Zakrzewski, G. A. Voth, P. Salvador, J. J. Dannenberg, S. Dapprich, A. D. Daniels, Ö. Farkas, J. B. Foresman, J. V. Ortiz, J. 
Cioslowski, D. J. Fox Gaussian 09 Revision A.1, Wallingford, 2009, Available at: http://www.gaussian.com.

29. M. W. Schmidt, K. K. Baldridge, J. A. Boatz, S. T. Elbert, M. S. Gordon, J. H. Jensen, S. Koseki, N. Matsunaga, K. A. Nguyen, S. Su, T. L. Windus, M. Dupuis, J. A. Montgomery, J. Comput. Chem. 1993, 14, 1347-1363.

30. M. Valiev, E. J. Bylaska, N. Govind, K. Kowalski, T. P. Straatsma, H. J. J. Van Dam, D. Wang, J. Nieplocha, E. Apra, T. L. Windus, W. A. de Jong, Comput. Phys. Commun. 2010, 181, 1477-1489.

31. D. R. Glowacki, C.-H. Liang, C. Morley, M. J. Pilling, S. H. Robertson, J. Phys. Chem. A 2012, 116, 9545-9560.

32. R. G. Gilbert, S. C. Smith, M. J. T. Jordan UNIMOL program suite (calculation of fall-off curves for unimolecular and recombination reactions), School of Chemistry, Sydney University, NSW 2006, Australia, 1993, Available at: ftp.chem.usyd.edu.au. 33. S. J. Klippenstein, A. F. Wagner, S. H. Robertson, R. Dunbar, D. M. Wardlaw Variflex software, 1.0 1999, Available at: http://chemistry.anl.gov/variflex.

34. V. Mokrushi ChemRate program suite, 1.5.2 2006, Available at: http://www.mokrushin.com/ChemRate/chemrate.html.

35. W. T. Duncan, R. L. Bell, T. N. Truong, J. Comput. Chem. 1998, 19, 1039-1052.

36. T. Yu, J. Zheng, D. G. Truhlar, Chem. Sci. 2011, 2, 2199-2213.

37. T. Yu, J. Zheng, D. G. Truhlar, J. Phys. Chem. A 2011, 116, 297-308.

38. G. Reinisch OpenSOAMS, a C++ library for the Statistics Of Atomic and Molecular Systems, 2012, Available at: http://opensoams.googlecode.com/svnhistory/r2/doc/html/index.html.

39. A. Miyoshi GPOP software, rev. 2013.07.15 2013, Available at: http://www.frad.t.u-tokyo.ac.jp/ miyoshi/gpop/. 


\section{Captions}

\section{$\underline{\text { Tables }}$}

Table 1. Contributions to the partition functions and to the thermodynamic properties of ideal gases.

Table 2. Comparison of KiSThelP capabilities with those of seven programs among the most widely used in the field of computational kinetics.

\section{Figures}

Figure 1. Example of KiSThelP input file content divided into seven sections.

Figure 2. Output produced by KiSThelP showing statistical and thermodynamic properties of a molecule obtained from electronic structure data; temperature and pressure parameter fields are visible at the top and bottom; electronic structure data can be change in fields on left side. [Color figure can be viewed in the online issue, which is available at wileyonlinelibrary.com]

Figure 3. Molecular statistical and thermodynamic calculations; 2D-plot can be displayed for selected properties (buttons on right side); KiSThelP enables to change input data and temperature and pressure parameters directly through the GUI and to visually probe how it affects results. [Color figure can be viewed in the online issue, which is available at wileyonlinelibrary.com]

Figure 4. Full report for reacting species as well as TST kinetic results; when a VTST calculation is invoked, the bottom panel shows a comparison between potential energy profile and the free energy profile. [Color figure can be viewed in the online issue, which is available at wileyonlinelibrary.com].

Figure 5. Logarithmic representation of rate constants against inverse temperature; prediction at four levels of theory (TST, VTST, TST/Tunnelling, VTST/Tunnelling); statistical fittings can be accessed from "Arrhenius" button (on right side); graph customization is possible ( $\mathrm{x}$ and y ranges, ...). [Color figure can be viewed in the online issue, which is available at wileyonlinelibrary.com] 
Figure 6. Full report of a RRKM simulation; data and parameters that were used to run the calculation are displayed (Lennard-Jones parameters, partition functions, thermal rate constants, high- and low-pressure limits, reaction properties, micro-canonical rate constants); energy threshold $\mathrm{E}_{0}$, collision efficiency, reaction path degeneracy can be changed interactively (left panel). [Color figure can be viewed in the online issue, which is available at wileyonlinelibrary.com]

Figure 7. 2D-plot of the RRKM falloff behaviour interactively obtained as a function of pressure $\mathrm{P}$ (bottom panel); numerical results as well as image can be saved on files. [Color figure can be viewed in the online issue, which is available at wileyonlinelibrary.com] 
Table 1. Contributions to the partition functions and to the thermodynamic properties of ideal gases.

\section{Rotation}

\begin{tabular}{|c|c|c|c|c|c|}
\hline & Translation & Vibration & Linear & Nonlinear & Electronic \\
\hline $\mathrm{Q}^{\mathrm{a}}$ & $\mathrm{Q}_{\text {trans }}=\left(\frac{2 \pi \mathrm{mk}_{\mathrm{b}} \mathrm{T}}{\mathrm{h}^{2}}\right)^{3 / 2} \frac{\mathrm{RT}}{\mathrm{P}}$ & $\begin{array}{c}\mathrm{Q}_{\mathrm{vib}}^{\mathrm{v}=0}=\prod_{\mathrm{i}} \frac{1}{1-\mathrm{e}^{-\theta_{\mathrm{i}} / \mathrm{T}}} \\
\theta_{\mathrm{i}}=\mathrm{h} v_{\mathrm{i}} / \mathrm{k}_{\mathrm{b}}\end{array}$ & $\begin{array}{l}Q_{r o t}=\frac{T}{\sigma_{r} \theta_{r}} \\
\theta_{r}=\frac{h^{2}}{8 \pi^{2} I k_{b}}\end{array}$ & $\begin{array}{c}\mathrm{Q}_{\text {rot }}=\frac{\pi^{1 / 2}}{\sigma_{\mathrm{r}}}\left(\frac{\mathrm{T}^{3}}{\theta_{\mathrm{a}} \theta_{\mathrm{b}} \theta_{\mathrm{c}}}\right)^{1 /} \\
\theta_{\mathrm{x}}=\frac{\mathrm{h}^{2}}{8 \pi^{2} \mathrm{I}_{\mathrm{x}} \mathrm{k}_{\mathrm{b}}}\end{array}$ & $Q_{\text {elec }}=g_{\text {elec }}^{\text {ground }}$ \\
\hline$U^{b}$ & $\mathrm{U}_{\text {trans }}=\frac{3}{2} \mathrm{RT}$ & $\mathrm{U}_{\mathrm{vib}}^{\mathrm{v}=0}=\mathrm{R} \sum_{\mathrm{i}} \frac{\theta_{\mathrm{i}}}{\mathrm{e}^{\theta_{i} / \mathrm{T}}-1}$ & $\mathrm{U}_{\mathrm{rot}}=\mathrm{RT}$ & $\mathrm{U}_{\mathrm{rot}}=\frac{3}{2} \mathrm{RT}$ & $\mathrm{U}_{\text {elec }}=0$ \\
\hline $\mathrm{S}^{\mathrm{c}}$ & $S_{\text {trans }}=R\left(\ln Q_{\text {trans }}+\frac{5}{2}\right)$ & $\begin{array}{c}\mathrm{S}_{\mathrm{vib}}=\mathrm{R} \ln \mathrm{Q}_{\mathrm{vib}}^{\mathrm{v}=0}+ \\
\frac{\mathrm{U}_{\mathrm{vib}}^{\mathrm{v}=0}}{\mathrm{~T}}\end{array}$ & $\mathrm{~S}_{\mathrm{rot}}=$ & $R \ln Q_{\text {rot }}+\frac{U_{\text {rot }}}{T}$ & $\mathrm{~S}_{\text {elec }}=\mathrm{R} \ln \mathrm{g}_{\text {elec }}^{\text {ground }}$ \\
\hline$C_{v}^{d}$ & $\mathrm{C}_{\mathrm{v}, \text { trans }}=\frac{3}{2} \mathrm{R}$ & $\mathrm{V}_{\mathrm{v}, \mathrm{vib}}=\mathrm{R} \sum_{\mathrm{i}} \frac{\left(\theta_{i} / \mathrm{T}\right)^{2} \mathrm{e}_{\mathrm{i}}^{\theta_{i} / \mathrm{T}}}{\left(\mathrm{e}^{\theta_{i} / \mathrm{T}}-1\right)^{2}}$ & $\mathrm{C}_{\mathrm{v}, \text { rot }}=\mathrm{R}$ & $\mathrm{C}_{\mathrm{v}, \mathrm{rot}}=\frac{3}{2} \mathrm{R}$ & $\mathrm{C}_{\mathrm{v}, \mathrm{rot}}=0$ \\
\hline
\end{tabular}

${ }^{\mathrm{a}}$ Individual partition functions, ${ }^{\mathrm{b}}$ Internal thermal energies (thus, without ZPE vibrational contribution), ${ }^{\mathrm{c}}$ Entropies, ${ }^{\mathrm{d}}$ heat capacity at constant volume

Table 2. Comparison of KiSThelP capabilities with those of seven programs among the most widely used in the field of computational kinetics.

\begin{tabular}{|c|c|c|c|c|c|c|c|c|}
\hline & KiSThelP & POLYRATE & ChemRate & TheRate & VariFlex & UNIMOL & MultiWell & MesMER \\
\hline GUI & - & & - & - & & & & \\
\hline $\begin{array}{l}\text { Typical } \\
\text { processus }\end{array}$ & Uni/Bi/Eq & Uni/Bi & Uni & Uni/Bi & Uni/Bi & Uni & Uni & Uni \\
\hline $\begin{array}{l}\text { Multidim. } \\
\text { tunnelling }\end{array}$ & & - & & - & & & - & \\
\hline RRKM & - & & - & & - & - & - & - \\
\hline $\begin{array}{l}\text { Parse } \\
\text { CCS } \\
\text { output }\end{array}$ & $\begin{array}{c}\text { Gaussian, } \\
\text { NWChem, Gamess }\end{array}$ & $\begin{array}{c}\text { Gaussian, NWChem, } \\
\text { Gamess, ... }\end{array}$ & & Gaussian & & & Gaussian & \\
\hline $\begin{array}{l}\mathrm{ME}^{\mathrm{a}} / \text { multiple } \\
\text { channels }\end{array}$ & & & - & & - & - & - & - \\
\hline $\begin{array}{l}\text { Language } \\
\text { Platforms }\end{array}$ & $\begin{array}{c}\text { Java } \\
\text { W/L/M }\end{array}$ & $\begin{array}{l}\text { Fortran } \\
\text { W/L/M }\end{array}$ & W & $\begin{array}{c}\text { Java } \\
\text { W/L/M }\end{array}$ & $\begin{array}{l}\text { Fortran } \\
\text { W/L/M }\end{array}$ & $\begin{array}{c}\text { Fortran } \\
\text { W/L/M }\end{array}$ & $\begin{array}{c}\text { Fortran } \\
\text { W/L/M }\end{array}$ & $\begin{array}{c}\mathrm{C}++ \\
\mathrm{W} / \mathrm{L} / \mathrm{M}\end{array}$ \\
\hline
\end{tabular}

\footnotetext{
${ }^{\mathrm{a}}$ Master-Equation treatment
} 
*MASS (in uma)

18.01056

Figure 1

*END

*FREQUENCIES (in cm-1)

1682.8470

3892.0332

4029.7598

*END

*LINEAR

not linear

*END

* NUMBER OF SYMMETRY

1

*END

*MOMENT OF INERTIA (in au)

2.25323

4.11640

6.36963

*END

*POTENTIAL ENERGY (in hartree)

$-76.219785478469$

*END

*ELECTRONIC DEGENERACY

1

* END 
Figure 2

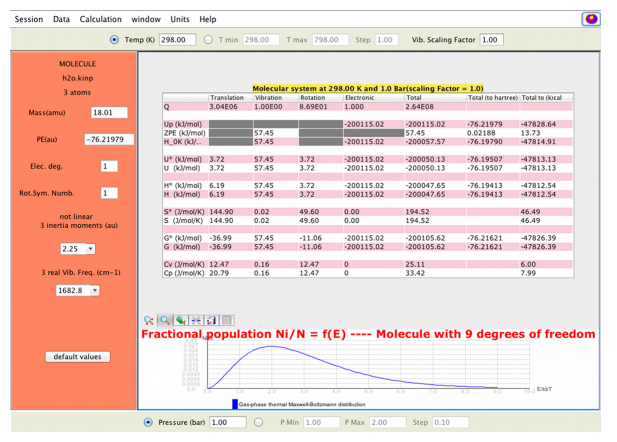


Figure 3

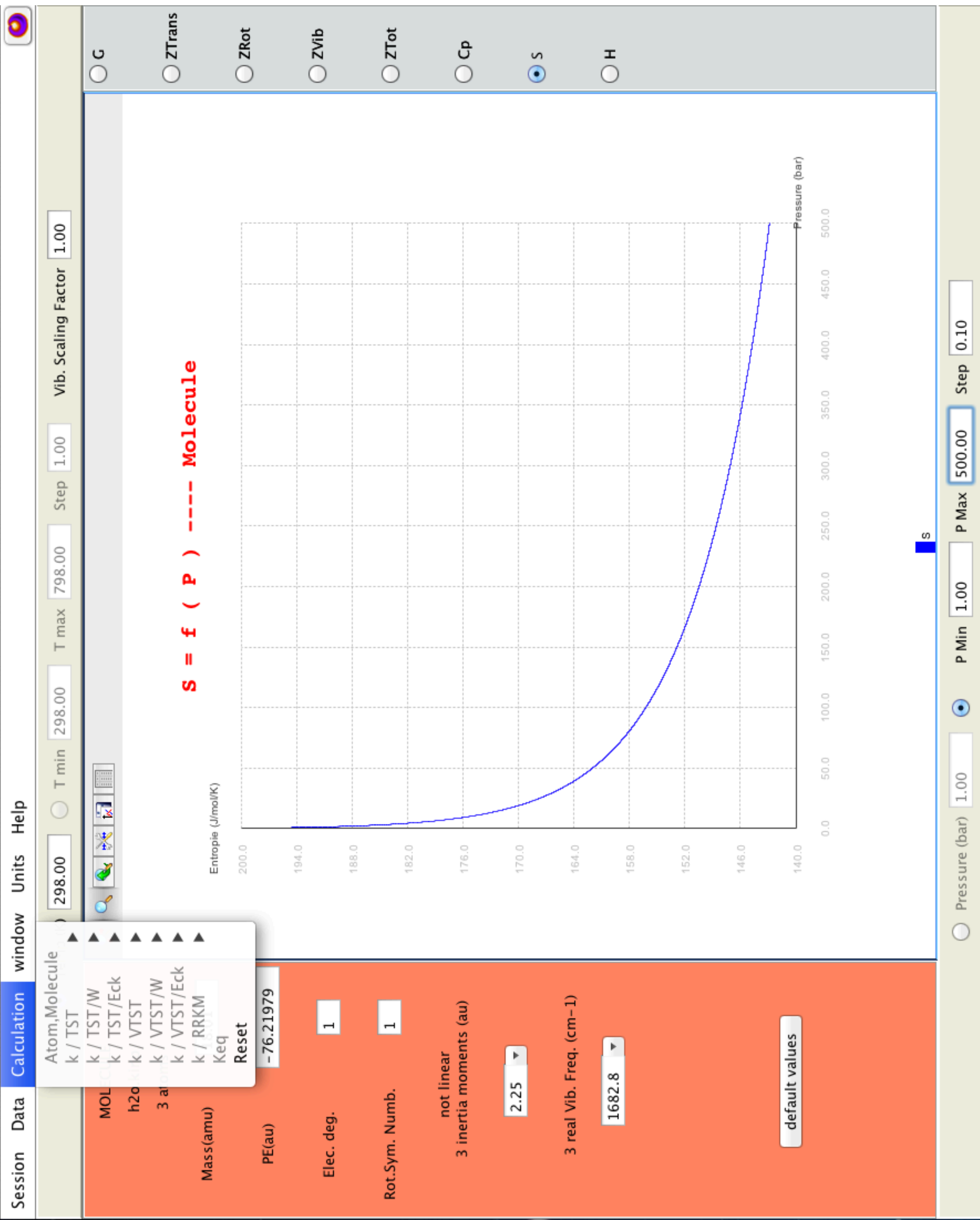


Figure 4

Session Data Calculation window Units Help

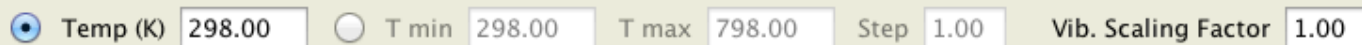

\section{BIMOLECULAR REACTION}

DATA FILENAMES :

h.kinp

ch4.kinp

reactionPath.kinp

Reaction path degeneracy :

Re

1

Bimolecular reaction at $298.0 \mathrm{~K}$ and 1.0 Bar(scaling Factor $=1.0-$ reaction path degeneracy $=1$ ) Dividing surface position that maximizes $\Delta \mathrm{G}^{\circ}: 0.1$

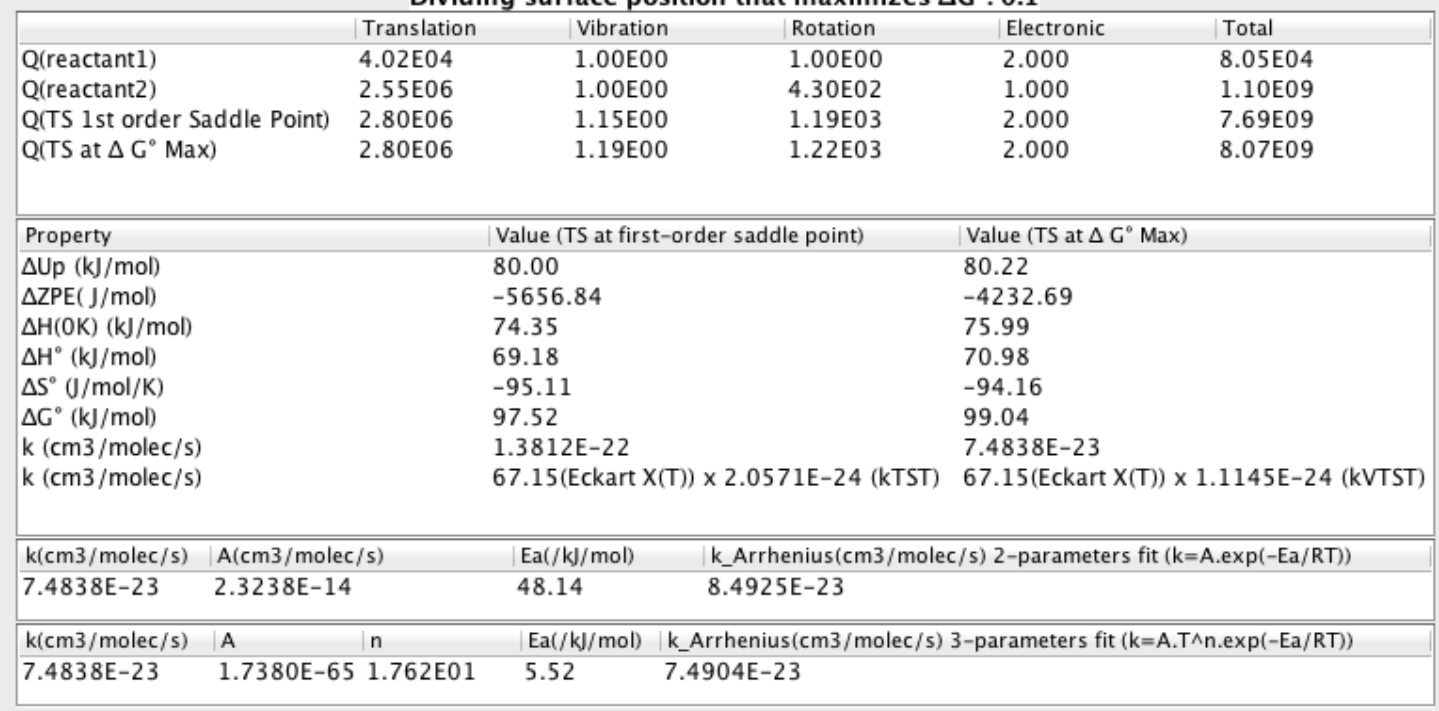

KINETIC LEVEL :

vtst_eck

$\Delta \mathrm{H}_{\mathrm{rev}}^{0^{-}}(\mathrm{OK})=50.0\left(\mathrm{~kJ} \cdot \mathrm{mol}^{-1}\right)$

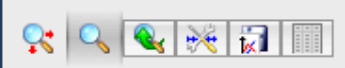

Activation energy profile $=\mathbf{f ( I R C )}$----

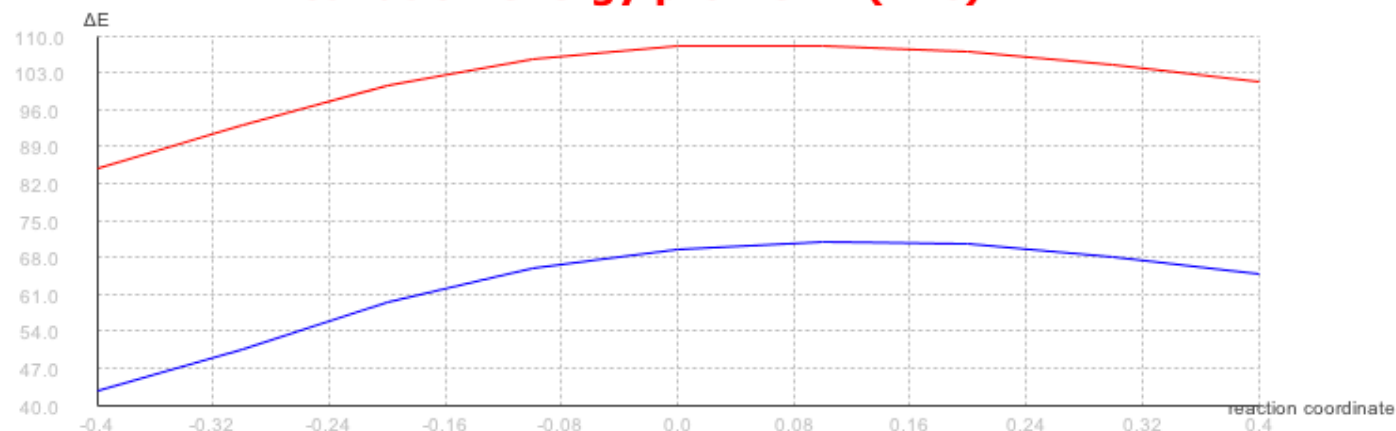

\begin{tabular}{l|l}
$\Delta \mathrm{E}$ & $\Delta \mathrm{G}^{\circ}(\mathrm{T})$
\end{tabular}

$\begin{array}{lllll}- \text { Pressure (bar) } & 1.00 & \text { P Max } & 500.00 & \text { Step } 0.10\end{array}$ 
Figure 5

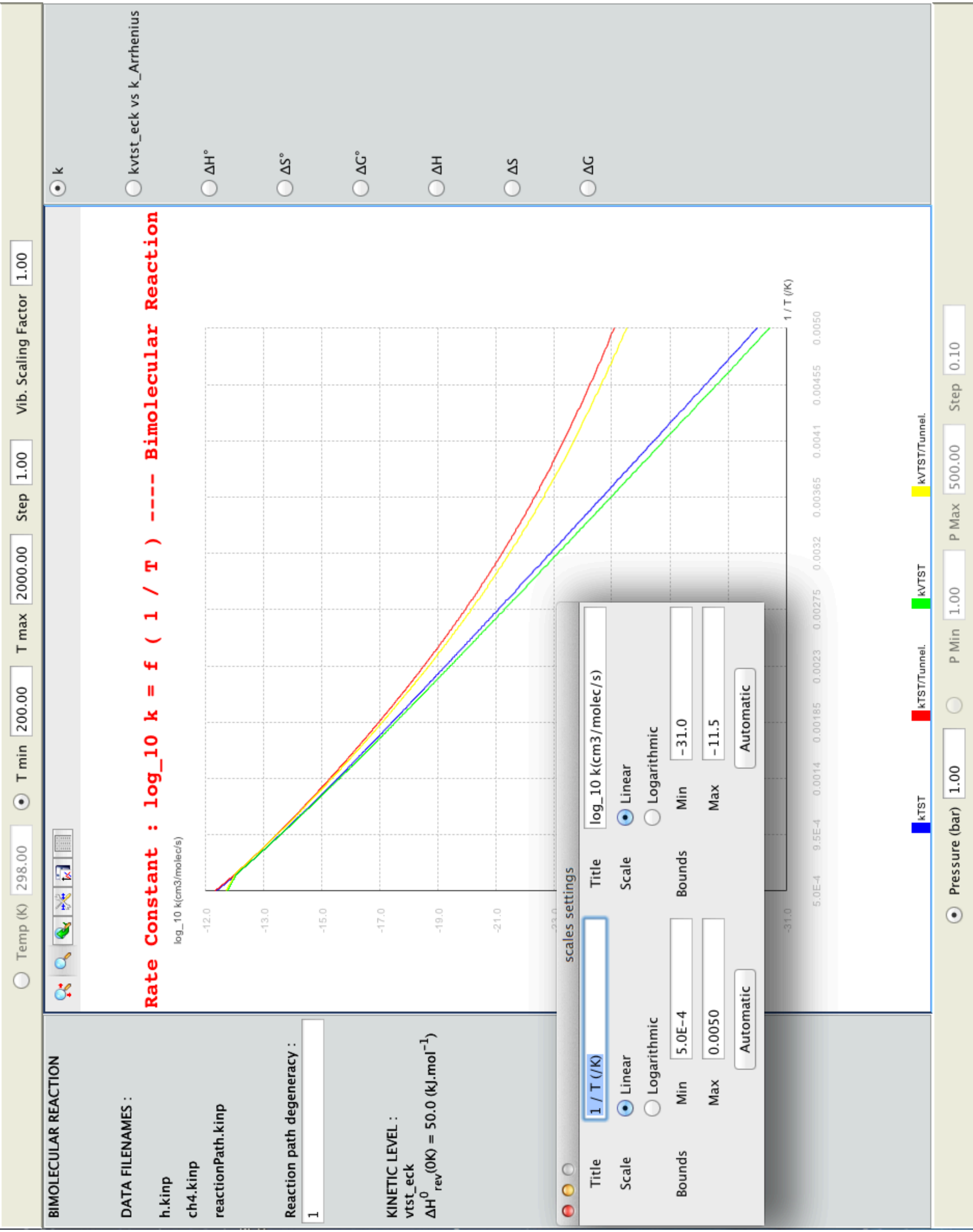


Figure 6

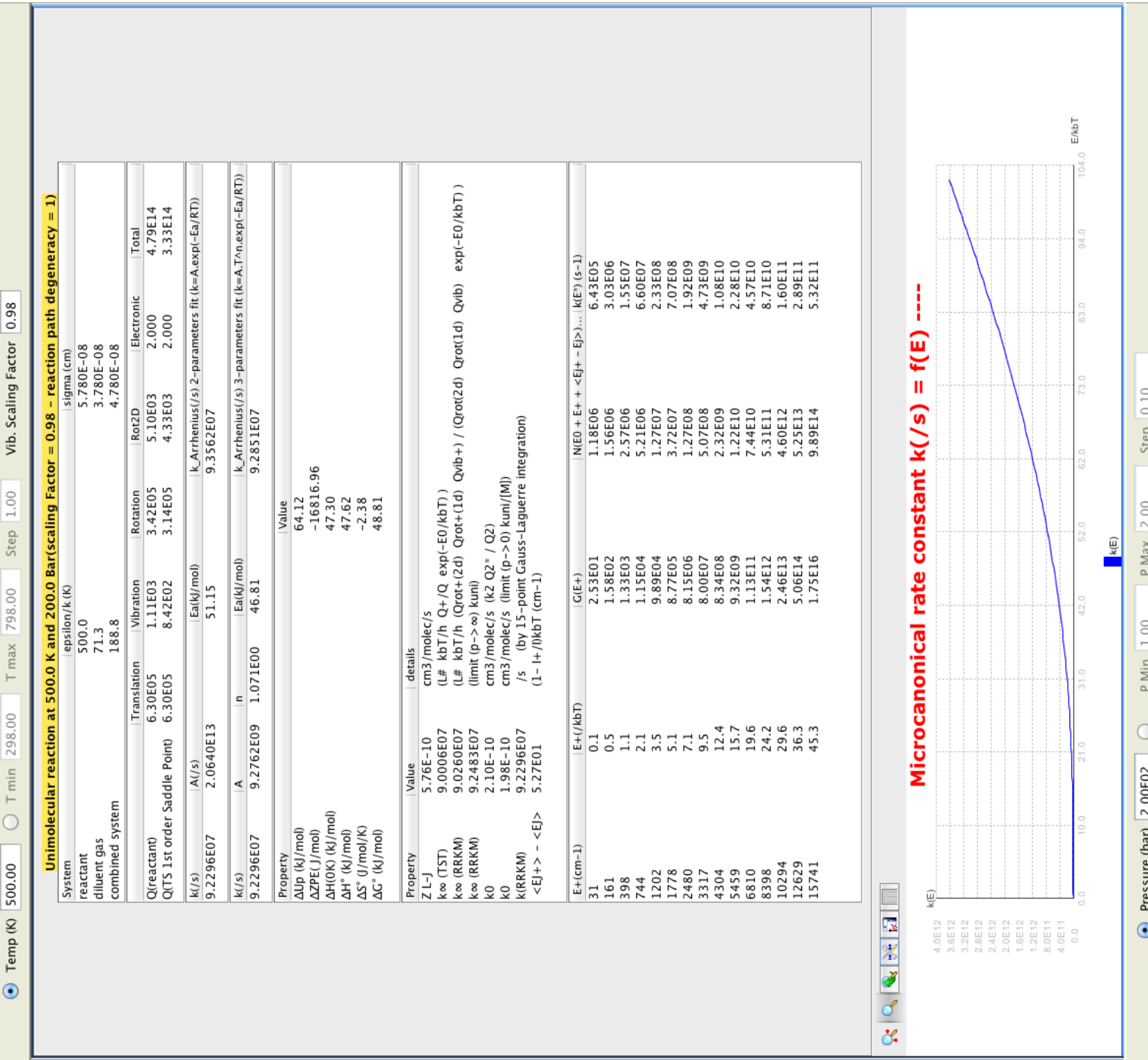


